\title{
Ambiente, adaptación y estrés
}

\author{
Koscinczuk, P. \\ Cátedra de Patología Médica, Facultad de Ciencias Veterinarias, Universidad Nacional del Nordeste, Sargento \\ Cabral 2139, Corrientes (3400). Argentina. Tel/Fax. 03783-425753. E-mail: pkoscinczuk@hotmail.com
}

\begin{abstract}
Resumen
Koscinczuk, P.: Ambiente, adaptación y estrés. Rev. vet. 25: 1, 67-76, 2014. El bienestar y la supervivencia de los individuos se ven amenazados por cambios ambientales; para restablecer la homeostasis y lograr adaptarse, el organismo pone en marcha respuestas colectivas de estrés. Estas respuestas están mediadas por circuitos interconectados del sistema límbico frontal, hipotálamo y cerebro, donde la liberación final de glucocorticoides asegura el aporte de energía mediante movilización de glucosa. El resultado final de la sobreexposición a glucocorticoides se refleja en la alteración de los sistemas corporales con hiperglucemia, hipertensión, infecciones recurrentes, alteración de la memoria y del control del comportamiento. Reconocer los eventos que pueden estresar a los animales domésticos no es sencillo. Mientras que algunos desencadenantes de estrés son fácilmente reconocidos por el hombre (temperaturas extremas, falta de alimentos, lesiones severas) otros pueden pasar desapercibidos (territorio, relaciones sociales, motivaciones, olores y sonidos). Por otra parte, se reconoce que la habituación a la especie humana en edad temprana es indispensable para una buena relación humano-animal, más aún, el ambiente humano puede ser percibido como un factor estresante en sí mismo.
\end{abstract}

Palabras clave: animales, salud, bienestar, sufrimiento, cognición.

\begin{abstract}
Koscinczuk, P.: Environment, adaptation and stress. Rev. vet. 25: 1, 67-76, 2014. Animal welfare and the survival of a particular species are threatened by environmental changes; for the reestablishment of homeostasis and adaptation, organisms put on collective stress responses. These responses are mediated by interconnected circuits of the frontal limbic system, hypothalamus and brain, where the final release of glucocorticoids guarantee energy supply through glucose mobilization, thus affecting brain reward and learning processes. Stress becomes detrimental when the animal is not able to neutralize the stressor, real or perceived. Consequences of over exposition to glucocorticoids are characterized by hyperglycemia, hypertension, recurrent infections and memory, as well as behavior modification. Determination of animal stressors can be complicated. While some stressors are easily recognized by human beings (extreme temperatures, lack of food, severe injuries), others are not (territory, social relationships, motivation, odors and sounds). On the other hand, habituation to humans at an early age could be indispensable to reach a good human-animal relationship, as human environment could be perceived as a stressor itself. In the particular case of domestic animals it is considered that habituation must begin as soon as possible to reach a good human animal relationship. People in daily contact with animals, such as veterinarians, are in the best position to recognize suffering and give advice to owners for the environmental modifications that may improve animal welfare for a particular species.
\end{abstract}

Key words: animals, health, welfare, suffering, cognition.

\section{INTRODUCCIÓN}

Los animales domésticos, sean de compañía (perros y gatos), de producción (bovinos, ovinos, caprinos, camélidos, cerdos, aves) o de trabajo (equinos, burros, bueyes), diariamente se enfrentan a estímulos ambientales, dentro de los cuales se debería incluir al hom-

Recibido: 12 febrero 2014 / Aceptado: 3 abril 2014 bre $^{37,61}$. Estos cambios alteran la homeostasis afectando no sólo el bienestar sino también la supervivencia de los individuos y de las especies. Las respuestas de estrés generadas para hacer frente a estos cambios, permiten mantener el delicado equilibrio de los diferentes órganos y sistemas y supone la activación coordinada del sistema nervioso autónomo y neuroendocrino ${ }^{58}$. Su finalidad es re-establecer la homeostasis y permitir la adaptación del individuo ${ }^{8,35,58}$. Dentro de estas res- 
puestas se observan cambios de comportamiento que podrían ser interpretados como un intento de optimizar las respuestas de adaptación, por la cual los animales procuran alcanzar estados emocionales positivos y evitan el sufrimiento, aumentando las probabilidades de supervivencia ${ }^{21,22,61}$.

El concepto de estrés como fenómeno biológico ha sufrido variaciones a lo largo del tiempo. Walter Cannon y sus colaboradores a principios del siglo XX describieron la actividad del sistema nervioso simpático y la llamaron respuesta de "fight or flight" (pelear o escapar). Recién alrededor de 1950, Hans Selye popularizó el concepto de estrés asociado al síndrome general de adaptación, reconociendo que además del sistema nervioso central había otros sistemas corporales involucrados, como las glándulas pituitaria y adrenal. Consideró tres etapas: fase de alarma (sin adaptación), de resistencia (adaptación) y de agotamiento (cuando el daño es tan intenso o prolongado que produce una pérdida del estado de salud o la muerte del individuo). Recién en la década de 1970, Selye ${ }^{50}$ sugirió que el estrés era la consecuencia biológica de la exposición a un ambiente adverso. Sin embargo, estos conceptos nada dicen respecto de la naturaleza de los factores estresantes y tampoco explican porqué las respuestas no siempre son coherentes en intensidad y amplitud, variando considerablemente entre individuos ${ }^{8}$.

Las alteraciones fisiológicas, conductuales e inmunológicas que se producen se conocen en su conjunto como respuestas de estrés. Presentan variaciones incluso entre individuos de una misma especie y están influidas por el estado fisiológico, edad, sexo, experiencias previas y por la capacidad de controlar y/o predecir la situación ${ }^{35}$. Las respuestas están mediadas por grandes circuitos interconectados en el sistema límbico frontal, hipotálamo y cerebro, relacionados con la memoria y la recompensa ${ }^{58}$. Por esto, resulta adecuado considerar que el estrés es la respuesta biológica generada cuando un animal percibe una amenaza a su homeostasis ${ }^{35}$. Al igual que Selye, el modelo presentado por Moberg, divide la respuesta de estrés en tres momentos: reconocimiento del agente estresante, defensa biológica contra el mismo y sus consecuencias ${ }^{35}$. Sólo cuando la intensidad o la duración de la respuesta alteren las funciones fisiológicas o conductuales, el estrés fisiológico o euestrés se transformaría en estrés patológico o diestrés ${ }^{8,35}$.

En sí mismo, el estrés no es algo indeseable; son sus consecuencias las que pueden ser buenas o malas para el organismo. Si consideramos al estrés como un concepto complementario al bienestar ${ }^{59}$, entender los mecanismos subyacentes nos permitirían ser más objetivos al momento de evaluar la calidad de vida de los animales.

A continuación describiremos cómo se produce la integración de los ejes simpático adreno medular (SNA) e hipotálamo pituitario adrenal (HPA) en el hipotálamo, teniendo en cuenta la acción coordinada de las denominadas hormonas de estrés. Comenzaremos con las res- puestas rápidas de estrés mediadas por el SNA y luego por el HPA, describiendo de qué manera la estimulación intensa o prolongada en el tiempo puede afectar la salud. Finalmente, detallaremos algunos de los factores desencadenantes de estrés que diariamente pueden ser percibidos por los animales domésticos. Identificarlos $\mathrm{y}$ tomar medidas para minimizar los efectos negativos que pudieran generar, permitirían mejorar el bienestar.

\section{EJE SIMPÁTICO ADRENOMEDULAR}

El SNA es el responsable de la respuesta inmediata luego de una disrupción de la homeostasis o amenaza al bienestar del individuo. Su activación se puede producir tanto por aquellos estímulos que son percibidos por los órganos de los sentidos (receptores exteroceptivos) como por aquellos receptores que se encuentran dentro del organismo (receptores interoceptivos) como por ejemplo, los preso y baro receptores del arco aórtico 26,58 .

La exposición a cualquier factor estresante lleva a la activación de neuronas pre-ganglionares simpáticas de las células intermedio laterales de la columna toracolumbar y de la médula espinal que van desde T1 a L2. Estas neuronas preganglionares se proyectan a los ganglios pre o paravertebrales y desde allí, mediante la acción de la noradrenalina, estimulan a los diferentes órganos efectores. Dentro de estos últimos se incluyen las células cromafines de la médula adrenal. El aumento en la concentración de noradrenalina (desde los nervios simpáticos) y adrenalina (por estimulación directa de la médula adrenal) tienen como finalidad aumentar el flujo de sangre al sistema nervioso central y al sistema músculo esquelético, cuyos órganos deberán coordinar una respuesta efectiva para controlar al agresor percibido ${ }^{26,58}$.

Esta reacción de alarma mejora la frecuencia y fuerza de contracción del corazón, aumentando el volumen minuto, que junto a la vasoconstricción periférica generan un aumento de la presión arterial. Por otra parte, el bazo efectúa esplenocontracción cediendo a la circulación general un cupo adicional de glóbulos rojos. Al mismo tiempo, se produce el aumento de la frecuencia respiratoria y la relajación de los bronquiolos para optimizar la hematosis, favoreciendo el intercambio de oxígeno $\left(\mathrm{O}_{2}\right)$, a nivel pulmonar. En todos los casos, la meta final es mejorar el aporte de sangre oxigenada al cerebro y al sistema musculoesquelético ${ }^{25,48}$.

El aporte de $\mathrm{O}_{2}$ a todas las células de la economía mejora tanto la eficiencia metabólica como la capacidad de trabajo. Mediante el metabolismo aeróbico, por cada molécula de glucosa se obtienen 38 moléculas de trifosfato de adenosina (ATP). Cuando falta $\mathrm{O}_{2}$, las células deben recurrir a la vía anaeróbica donde, por cada molécula de glucosa sólo se generan 3 moléculas de ATP más lactato ${ }^{48}$. Para que las células puedan cumplir con sus funciones plásticas, secretoras o reproductoras, deben tener una producción óptima de ATP, que depende de la disponibilidad de $\mathrm{O}_{2}$ y de su capacidad para uti- 
lizarlo. Tanto la glucosa como el $\mathrm{O}_{2}$ son transportados por la sangre y desde allí al espacio extracelular y a las células. El mantenimiento del volumen intravascular y el gasto cardíaco se tornan indispensables para garantizar la distribución de $\mathrm{O}_{2}$, mientras que el sistema respiratorio realizará los procesos de captación a nivel alveolar ${ }^{48}$. Algo muy interesante es que, cuando se incrementa el metabolismo basal aumenta la temperatura corporal, con un desplazamiento hacia la derecha de la curva de disociación oxígeno-hemoglobina, lo que favorece el pasaje de $\mathrm{O}_{2}$ a los tejidos ${ }^{25}$.

Si bien la activación del sistema nervioso simpático es adaptativa, tiene un alto costo energético y la hipertensión arterial generada aumenta la morbimortalidad. Para evitar y controlar sus posibles efectos nocivos es que a continuación se produce una activación del sistema nervioso parasimpático. Este mecanismo de dos pasos, simpático en segundos y parasimpático en minutos, asegura un episodio secretorio amplificado y prolongado ${ }^{58}$. La estimulación parasimpática así generada, opuesta a la del simpático, ejerce una acción moduladora, con disminución de la frecuencia cardíaca. Esta respuesta también se asocia a otros efectos, como hipersecreciones digestivas (hipersalivación y diarrea), micción espontánea y miosis ${ }^{25,58}$.

\section{EJE HIPOTÁLAMO PITUITARIO ADRENAL}

La activación del SNA no se produce en forma aislada, sino que se relaciona estrechamente con la estimulación del eje HPA y los glucocorticoides (GC), de esta manera el organismo se asegura un aporte adicional de energía mediante la movilización de glucosa. Esta fuente de energía adicional permitiría un trabajo más eficiente de las células, reforzando y mejorando la respuesta inicial que había comenzado con la activación del sistema simpático ${ }^{25}$.

La activación del eje HPA se produce pocos segundos después de la del SNA y comienza con la estimulación del núcleo paraventricular del hipotálamo. No se conoce en profundidad si esta estimulación se produce directamente por medio de la noradrenalina de terminales nerviosas del SNA, cuyas prolongaciones alcanzan el hipotálamo, o bien mediante la adrenalina liberada a la circulación general desde la médula adrenal ${ }^{58}$. Lo importante es que, en el hipotálamo, el soma de las neuronas parvocelulares se encarga de sintetizar dos neuropéptidos: hormona liberadora de corticotropina (CRH) y vasopresina, que desde allí, y dentro de la misma neurona, son trasladados hacia la eminencia media y al tuber cinereum (prolongación del tejido hipotalámico dentro del tallo hipofisario) ${ }^{55,58}$. Cuando se produce la estimulación hipotalámica, se desencadena la libración de estos neuropéptidos al sistema portal hipotálamo-hipofisario. Desde allí se dirigen directamente a las células cromatófobas de la adenohipófisis estimulando la producción de péptidos derivados de la pro-opiomelanocortina: $\mathrm{ACTH}, \beta$-endorfina y $\alpha$ - melanocitoestimulante ${ }^{55}$. La CRH, necesaria para estimular a la adenohipófisis, no sólo se sintetiza en el hipotálamo, sino también en otros numerosos sitios del cerebro incluyendo amígdala, strias terminalis, corteza prefrontal y células alrededor del locus coeruleus ${ }^{39}$.

La ACTH actúa directamente sobre la corteza adrenal estimulando la producción de corticosteroides, principalmente GC y andrógenos ${ }^{35}$. Los GC son los responsables de ejercer el control de secreción de las hormonas de estrés mediante retroalimentación negativa sobre hipotálamo e hipófisis. Se ha demostrado que estos GC también son capaces de regular la secreción de $\mathrm{CRH}$, vasopresina y ACTH mediante retroalimentación negativa (vide supra) tanto en el cerebro (corteza pre-frontal) como en la hipófisis anterior ${ }^{36,55,63}$. Más adelante describiremos este mecanismo de acción, que involucra a dos tipos de receptores para GC.

Algunos autores consideran al núcleo paraventricular del hipotálamo como una pequeña central integradora ${ }^{58}$ que recibe información no sólo del sistema nervioso autónomo, sino también señales metabólicas de la corteza cerebral y de los órganos subfornicales, encargados de monitorear el plasma, así como también del sistema límbico, amígdala e hipocampo. En este último caso las señales recibidas están relacionadas con las emociones de los individuos y es donde el sistema cognitivo cumple un papel importante ${ }^{7,58}$. Es de destacar que el eje HPA puede ser estimulado por diversos desencadenantes: infecciones, trauma, dolor, y/o estímulos psíquicos, entre otros ${ }^{39}$.

\section{METABOLISMO DE LOS CORTICOSTEROIDES}

Todos los corticosteroides sintetizados por la corteza adrenal -GC, mineralocorticoides (MC) y andrógenos- tienen una estructura química semejante derivada del colesterol ${ }^{36}$. Pequeñas diferencias en su conformación permiten la activación de distintos receptores. Mientras que los GC tienen actividad sobre el metabolismo de los glúcidos (de allí su nombre), los MC participan en la regulación del equilibrio hidrosalino (grupo representado por la aldosterona) ${ }^{25}$.

El cortisol es el GC más activo del eje HPA en bovinos, ovinos, cerdos, zorrinos, zorros, peces ${ }^{36}$, felinos ${ }^{10}$, caninos ${ }^{6}$ y el ser humano ${ }^{55}$; mientras que la corticosterona lo es en aves y roedores de laboratorio ${ }^{36}$. En este trabajo, cada vez que mencionemos GC, haremos referencia a cortisol y corticosterona por ser los de mayor impacto en las respuestas de estrés.

La respuesta esteroideogénica a un pulso de ACTH comienza y termina en pocos minutos. La forma activa de esta hormona está presente solo unos 5 a 10 minutos, momento en el que se une a su receptor específico en la superficie de la célula cortical adrenal, activando la síntesis y liberación de cortisol a la circulación general ${ }^{25}$. El 90\% del cortisol circulante se une a una a2globulina fijadora de cortisol denominada transcortina; mientras que el $10 \%$ restante permanece libre. Esta pequeña fracción libre es la biológicamente activa, pu- 
diendo atravesar las membranas biológicas, incluso la barrera hematoencefálica y las membranas celulares: allí ejerce su acción y finalmente es metabolizada y/o eliminada ${ }^{25}$. En algunas situaciones la producción de cortisol sobrepasa la capacidad fijadora de la transcortina, haciendo que aumente la fracción libre, eliminándose fácilmente por orina ${ }^{53}$, materia fecal, saliva ${ }^{29} \mathrm{o}$ saturando los pelos del individuo ${ }^{2,14}$. Se ha demostrado que el cortisol así eliminado es proporcional al plasmático ${ }^{28}$ y su cuantificación resulta una herramienta útil para evaluar las respuestas endocrinas de estrés de manera no invasiva ${ }^{6,14,29,53}$.

El índice de declinación del cortisol es de 60-90 minutos. Este puede convertirse en cortisona, de menor efectividad biológica o ser metabolizado por el hígado. Su transformación a cortisona se realiza enzimáticamente mediante una reacción reversible. Esta reversibilidad explica, de algún modo, la actividad biológica atribuida a la cortisona, que también debería considerarse cuando se interpreta la retroalimentación negativa del cortisol sobre hipotálamo e hipófisis ${ }^{63}$. La inactivación final de ambos ocurre en el hígado, donde se transforman en tetrahidrocortisol y tetrahidrocortisona, biológicamente inactivos. Luego son conjugados con ácido glucorónico, convirtiéndose en hidrosolubles para ser eliminados por los riñones o la vesícula biliar.

\section{RECEPTORES CORTICOSTEROIDES}

Los GC, luego de ser liberados a la circulación, inician su interacción molecular primaria en células blanco a través de la unión a receptores nucleares. Se conocen dos tipos de receptores nucleares activados por cortisol: los receptores glucocorticoides (GRs) y los mineralocorticoides (MRs) ${ }^{25}$. Estos actúan como ligandos de factores de trascripción, modificando la amplitud, latencia y duración de la actividad biológica de los genes de transcripción ${ }^{49}$.

Cabe mencionar que hay dos mecanismos de acción de los GC: genómico y no genómico. Las acciones genómicas ocurren principalmente luego de la unión a los GRs, pero, en algunos tejidos, se pueden dar luego de la estimulación de MRs ${ }^{49}$. Por esta acción, las células modifican sus funciones plásticas, secretoras y metabólicas. Por otra parte, la respuesta no genómica que ocurre pocos minutos después de la liberación de los GC, se relaciona con el efecto de retroalimentación inhibitorio sobre el eje HPA. Esto ocurre muy rápidamente, tan rápido que se descarta la posibilidad de que sea una acción genómica tal como se la considera en el sentido tradicional, aunque los detalles del mecanismo de acción todavía no se conocen en su totalidad ${ }^{58}$.

Si bien la afinidad de los GC por los MRs es baja, debe ser tenida en cuenta cuando se producen aumentos sostenidos de GC en una respuesta de estrés crónico ${ }^{36,49,58}$, particularmente en aquellos tejidos que tienen un metabolismo hidroelectrolítico activo, como el riñón, glándulas salivares y colon. En estos tejidos, que deberían responder únicamente a la aldosterona, sus receptores están protegidos de la acción del cortisol por la enzima $11 \beta$ hidroxiesteroideo deshidrogenasa (11ßHSD), encargada de inactivar al cortisol in situ transformándolo en cortisona. Cabe recordar que la aldosterona no solo puede ser liberada por la corteza adrenal bajo la influencia del sistema renina-angiotensina, sino también por acción de la misma ACTH ${ }^{36}$.

Los receptores GRs y MRs se encuentran distribuidos en todas las células de la economía, incluyendo el sistema nervioso central. Se considera que, a este nivel, los MRs tienen diez veces más afinidad por GC que los GRs y están ocupados, incluso, por niveles muy bajos de GC circulantes, ejerciendo una retroalimentación negativa sobre el cerebro. No sólo los GRs se hallan ampliamente distribuidos en el cerebro y se relacionan con hipotálamo e hipófisis, sino también los MRs, que son abundantes tanto en hipocampo como septum lateral, amígdalas e hipotálamo, relacionándose con el ritmo circadiano ${ }^{18,49}$.

Es conveniente destacar aquí que los GC sintéticos, como prednisolona y dexametasona, entre otros, usualmente no se unen a los MRs e interactúan deficientemente con los GRs del sistema neuroendocrino, lo que altera la retroalimentación negativa. Si bien son ampliamente utilizados terapéuticamente, sustituyendo a GC endógenos en sus efectos genómicos, al no ejercer un efecto inhibitorio adecuado sobre hipotálamo e hipófisis pueden ocasionar la supresión de la producción de GC a nivel de la glándula adrenal ${ }^{49}$, pudiendo generar hiperadrenocorticismo.

\section{CONSECUENCIAS DEL ESTRÉS CRÓNICO: DIESTRÉS}

La respuesta fisiológica de estrés a corto plazo permite al animal hacer frente a un estímulo estresante, poniendo en marcha un patrón de comportamiento adecuado, al mismo tiempo que intenta resolver la demanda energética generada. De esta manera, el individuo se libraría del factor estresante que ha considerado una amenaza. Una vez controlado el efecto adverso, el sistema debería desactivarse y la respuesta de estrés desaparecer, lográndose la adaptación al evento estresante ${ }^{38}$. El estrés comienza a ser perjudicial -diestrés- cuando no se pueden poner en marcha mecanismos conductuales efectivos que logren neutralizar al desencadenante. Esto ocurre cuando el individuo no tiene control sobre la situación o no puede predecir lo que va a suceder. $\mathrm{Si}$ el estímulo estresante -real o imaginario- persiste y la respuesta "designada" para enfrentarlo permanece, el sistema neuroendocrino continúa a la expectativa ${ }^{11}$. Es interesante notar que -como ya se mencionara anteriormente- aún bajo las mismas circunstancias ambientales, algunos animales se estresan más fácilmente que otros debido a las diferencias individuales, determinadas por el temperamento propio del animal y la experiencia previa, entre otras muchas causas ${ }^{11,38}$. 
La falta de adaptación produce una estimulación excesiva y prolongada del eje HPA, con un aumento de $\mathrm{GC}^{32,41,49}$. Una excesiva elevación de los niveles de GC hacen que el costo de mantener la homeostasis sea muy alto para la economía ${ }^{32,58}$. El resultado final de esta sobreexposición a GC se podría ver reflejado en los sistemas que participan directamente en las respuestas de estrés y que a continuación se detallan: endocrino (hiperglucemia), circulatorio (hipertensión), cognitivo (alteraciones de la memoria y del control del comportamiento) e inmune (infecciones recurrentes).

Hiperglucemia: los GC promueven la gluconeogénesis, fenómeno que tiene lugar en el hígado y, en menor medida, en músculos y riñones. Simultáneamente aumentan el catabolismo proteico con disminución de la síntesis de proteínas y movilización asociada de ácidos grasos, produciendo hipercolesterolemia e hipertrigliceridemia ${ }^{54}$. Para garantizar que la glucosa liberada llegue al sistema nervioso y al corazón, el ritmo de utilización de glucosa por otras células de la economía se enlentece ${ }^{25}$. La hiperglucemia resultante no puede ser regulada correctamente por la insulina. En respuesta, el páncreas se ve superado en el intento de controlar la concentración de glucosa plasmática, llevando al agotamiento del órgano con alteración final del metabolismo de los glúcidos -hiperglucemia de estrés- ${ }^{23,27}$.

Hipertensión: el aumento sostenido de la presión arterial comienza con la liberación hipotalámica de hormona antidiurética (ADH) y la de aldosterona por parte de la corteza adrenal. Mientras que la ADH retiene agua, la aldosterona favorece la retención de sodio a nivel del líquido extracelular y elimina potasio a través de la orina, induciendo a la absorción osmótica de agua. Por otra parte, en el hipotálamo, el incremento de sodio plasmático estimula la ingesta de agua, resultando en un aumento de agua extracelular con muy poca variación de la natremia. Estos dos efectos combinados producen aumento del volumen sanguíneo e hipertensión a largo plazo ${ }^{25}$.

Alteraciones en la memoria y aprendizaje: los GC tienen importantes efectos sobre la estructura y función de algunas áreas del cerebro como el hipocampo y la amígdala. A corto plazo, la optimización del aporte de glucosa facilita el proceso de fijación y recuperación de la memoria. En cambio, concentraciones elevadas de GC a largo plazo pueden provocar daño a nivel del hipocampo, con atrofia neuronal asociada a una disminución de la habilidad de las células para utilizar glu$\operatorname{cosa}^{49}$. Esto afecta tanto a la capacidad de procesar información y tomar decisiones frente a nuevas situaciones desafiantes, como a la función integradora que cumple el hipocampo dentro de la regulación del eje HPA (hipótesis de la cascada de glucocorticoides-hiperactividad HPA) ${ }^{32}$.

Alteraciones en el control del comportamiento: se ha demostrado que la administración de $\mathrm{CRH}$ (directamente en el cerebro) o de ACTH en animales de laboratorio tienen efecto ansiogénico, acción que se asocia con respuestas de miedo, pánico o depresión ${ }^{39}$. Estos efectos, mediados por la amígdala y diferentes áreas de la corteza cerebral, se relacionan con la ocurrencia de diferentes comportamientos asociados a diestrés que oscilan desde el acicalamiento excesivo, hiperactividad, bulimia, respuestas exageradas a los estímulos ambientales, hasta los comportamientos de evasión ${ }^{39} \mathrm{y}$ supresión de los comportamientos exploratorios, ingestivos o reproductivos ${ }^{49}$.

Inmunosupresión: varios tipos de agentes estresantes -endo-exotoxinas, microorganismos inoculados, o factores psicológicos- producen la activación rápida del sistema inmune a través de citoquinas ${ }^{49}$. La liberación de GC ante la situación estresante tendrá por función modular la respuesta inflamatoria e inmune del animal. Esto se logra por dos mecanismos, bloqueando la liberación de mediadores químicos que activan el proceso inflamatorio (histamina, bradiquinina, enzimas proteolíticas, prostaglandinas y leucotrienos) y favoreciendo la desaparición de la respuesta inflamatoria, al actuar sobre los leucocitos ${ }^{25}$. Los GC alteran el tráfico y la función de las células periféricas, producen disminución de los niveles circulantes de linfocitos (más de los T que de los B), eosinófilos, basófilos, macrófagos y monocitos y aumento de neutrófilos. Al mismo tiempo las concentraciones elevadas de GC se asocian con atrofia del timo y, en menor grado, muerte por apoptosis de los precursores inmaduros de células T y B ${ }^{49}$.

Desde el punto de vista fisiológico debemos recordar que la respuesta inmune se divide en tres grandes grupos: una respuesta dada por los linfocitos B mediante la producción de inmunoglobulinas y anticuerpos (eficiente contra infecciones bacterianas), otra celular, dada por los linfocitos $\mathrm{T}$, íntimamente relacionada con la programación que ocurre en el timo (eficiente contra infecciones virales, parasitarias, rechazo de células tumorales y en procesos autoinmunes) y, por último, la barrera de los macrófagos y los polimorfonucleares, que actúan junto a los anticuerpos para liberar al organismo del ataque bacteriano ${ }^{60}$.

De acuerdo a la respuesta de las poblaciones de linfocitos $\mathrm{T}$, los individuos se pueden clasificar en dos categorías: la primera corresponde a aquellos cuyos linfocitos $\mathrm{T}$ progenitores se transformarán en linfocitos TH1, produciendo interferón gamma e IL-2, lo que garantiza una buena respuesta tisular contra los agentes infecciosos, especialmente intracelulares. En cambio, en la segunda categoría, los linfocitos $\mathrm{T}$ progenitores se dirigen hacia la vertiente $\mathrm{TH} 2$, con producción de anticuerpos, IL-4 e IL-10, lo que favorece la respuesta humoral. Por lo tanto, aquellos individuos con mayor respuesta $\mathrm{TH} 2$, tendrán más probabilidades de ser alérgicos; en cambio, aquéllos con mayor respuesta TH1 serán menos alérgicos, pero con mayor respuesta citotóxica. Probablemente el costo por tener una respuesta TH1 eficiente es el desarrollo de enfermedades autoinmunes como tiroiditis, diabetes, artritis reumatoide y lupus; en cambio, los animales con mayor respuesta $\mathrm{TH} 2$ son generalmente alérgicos ${ }^{60}$. 
La clave de la respuesta inmune del organismo frente a las agresiones es el balance entre las respuestas TH1 y TH2. Se propone que, bajo condiciones fisiológicas, los GC modelan la respuesta del sistema inmune de manera tal que los componentes superfluos o propensos a autoinmunidad se inhiban selectivamente ${ }^{49}$.

\section{DE LOS FACTORES ESTRESANTES}

En este punto, deberíamos definir que un factor estresante puede ser tanto un desafío físico real a la homeostasis (cambios repentinos en la temperatura ambiente, restricción física, peleas) o la percepción de una amenaza (la mirada directa de un individuo dominante) ${ }^{37}$. No todos los desafíos impactan en los individuos de la misma manera ${ }^{15}$. Existen diversos factores que influyen sobre la capacidad de generar respuestas de estrés: edad ${ }^{24}$, herencia/historia genética ${ }^{40}$, sexo, gestación, lactancia y experiencias previas ${ }^{30}$, a los que se agregan otros factores como personalidad, entorno social y jerarquía social ${ }^{26}$. Cuando se evalúan animales domésticos, a esta suma de eventos, deberíamos agregar el significado que los animales le otorgan a los factores del ambiente en el cual viven ${ }^{13,59}$.

La domesticación puede ser interpretada como una forma especial de cambio evolutivo, por la cual una población de animales llega a adaptarse al hombre y su ambiente de cautiverio mediante cambios genéticos, entre otros ${ }^{44}$. Es indudable que dicho ambiente puede llegar a ser complejo y cambiante. Tal vez los desencadenantes de estrés más importantes en una población de animales en cautiverio sean aquellos sobre los cuales no tienen control y de los que no pueden escapar ${ }^{37}$. A continuación describiremos algunos ejemplos a tener en cuenta. Su identificación podría ser una herramienta útil para minimizar los efectos negativos que los mismos ejercen sobre el bienestar de estos animales.

\section{FACTORES AMBIENTALES ABIÓTICOS}

Olores: el olfato es uno de los sentidos más primitivos, todos los vertebrados lo utilizan para monitorear el ambiente ${ }^{37,52}$. Los receptores sensitivos para los olores, que se encuentran en la mucosa nasal, son neuronas cuyos axones se proyectan al bulbo olfatorio y desde allí al sistema límbico y al hipotálamo ${ }^{25,52}$. De esta manera, los olores no necesitan procesarse en la corteza superior para generar emociones y modificar la expresión de un comportamiento ${ }^{52}$. Existen señales olfatorias específicas producidas por feromonas, sustancias químicas secretadas en el ambiente por un individuo y percibidas por otro de la misma especie ${ }^{4}$. Muchos vertebrados han desarrollado un órgano independiente capaz de oler y degustar: el de Jacobson o vómero-nasal, situado debajo del paladar duro, comunicando las cavidades oral y nasal ${ }^{52}$. Cada especie produce y reconoce sustancias específicas cuya naturaleza química en algunos casos ha sido determinada. Pueden ser de estructura tan liviana como ésteres volátiles o tan pesadas como aminas cuaternarias ${ }^{4}$. Debido a que resultan esenciales en la comunicación y que asimismo permiten la interacción social y los comportamientos reproductivos ${ }^{37}$ muchas de ellas han sido sintetizadas artificialmente para su aplicación en distintos contextos, por ejemplo Feliway ${ }^{\circledR}$ (Ceva Santé Animale, France), análogo sintético de la feromona facial de gato ${ }^{20}$ o DAP (Dog Appeasing Pheromone, Ceva Santé Animale, France), utilizadas para el tratamiento de fobias o ansiedad en perros ${ }^{34,51,57}$, o las feromonas maternas equinas para el control de situaciones de miedo ${ }^{19}$. Sin dejar de lado la utilidad del uso de feromonas sintéticas, reconocer el significado que las feromonas naturales tienen en la comunicación social debería ser una de las metas para mejorar el bienestar, estimulando la presencia de feromonas de apaciguamiento y controlando o disminuyendo la presencia de aquellas feromonas asociadas a miedo, ansiedad, o desencadenantes de conductas agresivas $37,52,62$.

Sonidos: es un hecho que los seres humanos percibimos un rango muy limitado de sonidos (sólo entre 20 a $25.000 \mathrm{~Hz}$ ) y por lo tanto, la importancia que le otorgamos como estímulo resulta totalmente diferente al de los animales. Un ejemplo son los sonidos producidos por monitores, circuitos cerrados de cámaras y televisión, luces fluorescentes, que producen sonidos de alta frecuencia (más de $20 \mathrm{KHz}{ }^{37}$. Otro ejemplo lo constituyen camiones, bombas, filtros y otras máquinas que generan infrasonidos y vibraciones sísmicas ${ }^{37}$. Ambos extremos sonoros son detectados únicamente por otras especies de animales y no por seres humanos, afectándoles muchas veces su bienestar en contexto de encierro, sin que los hombres los tengan en cuenta.

Iluminación: en animales en confinamiento, la iluminación responde a necesidades humanas. En algunos sistemas productivos no se contempla el ritmo circadiano de los animales sino que pretenden modificarlo, con esquemas de iluminación donde se pueden producir situaciones extremas como lo son el exceso de luz artificial y el déficit de luz natural ${ }^{9}$. No cabe duda que las condiciones variables de luz alteran el sueño y el ritmo circadiano, generando más estrés al animal ${ }^{1}$.

Temperatura y humedad: en ambientes naturales los animales pueden recurrir a diversas estrategias para controlar su temperatura corporal: aguadas, sombras naturales, cavar pozos y otras. En cautividad estas estrategias están reducidas debido a que dependen de la distribución de recursos impartida por los seres humanos. El estrés térmico, tanto por bajas como por altas temperaturas, afecta el bienestar. En esta situación el control de la temperatura corporal se superpone con otras necesidades básicas, como alimentarse ${ }^{9}$. Además, se observó que el incremento de la temperatura corporal se asocia al aumento de las concentraciones de dopamina en el cerebro, generando una reducción de la exploración, afectando en consecuencia el comportamiento ingestivo y el sueño ${ }^{56}$.

Superficie de confinamiento: mientras que la restricción física o de movimientos es fácilmente recono- 
cida, generalmente la restricción social pasa desapercibida. En ambientes extensos el individuo puede conformar grupos sociales naturales; en cambio, en jaulas la conformación de los grupos sociales naturales resulta arbitraria o nula. Por este motivo, cuando se selecciona el tipo de jaula, sería relevante considerar no sólo la superficie de la misma con respecto al tamaño del individuo, sino también la superficie ideal mínima dentro de la cual convive el grupo social de esa especie, para permitirles a todos los individuos (o en su defecto a la mayoría del grupo) poder realizar los comportamientos de exploración, acicalamiento y juego. Asimismo, la posibilidad de realizar juego social mejora el bienestar y por lo tanto disminuye la posibilidad de diestrés. Unas pocas emociones positivas a lo largo del día tendrían mayor impacto sobre el bienestar que eliminar muchas negativas ${ }^{7}$.

Territorio/área de descanso/cama: la distribución espacial de los recursos es relevante. Por ejemplo, un gato elegirá como área de descanso un lugar alto y con visión amplia de todo su territorio, con una cama preferentemente blanda ${ }^{47}$. Muchas veces, no sólo es importante la ubicación o la superficie que se disponga para el descanso, sino también la posibilidad de superficie para otros comportamientos previos asociados. Por ejemplo, en granjas comerciales de gallinas ponedoras que disponen de nidos sobre-elevados, la presencia de plataformas más anchas frente a estos, facilita la expresión de comportamientos pre-postura ${ }^{31}$.

\section{FACTORES AMBIENTALES BIÓTICOS}

Alimentación: con frecuencia se habla de la importancia de la calidad de la materia prima de los alimentos, sin embargo los animales también se ven afectados de acuerdo a la forma en que se éstos se administran, teniendo en cuenta el tiempo, lugar y frecuencia de suministro, debido a que los seres humanos siguen el patrón conductual de su propia especie, sin considerar las necesidades de las demás. A lo largo de la evolución, las especies han desarrollado estrategias exploratorias e ingestivas que les permitieron adaptarse y sobrevivir. Como ejemplo podemos citar a dos carnívoros predadores: el perro y el gato. Mientras que el gato, cazador solitario, come presas pequeñas varias veces por día, los perros, en grupo, se alimentan de grandes presas una vez cada tanto ${ }^{32}$. Considerando esto, la forma correcta de administración de la ración diaria para gatos debería estar conformada por seis a ocho comidas pequeñas, la del perro tendría que ser una sola comida al día ${ }^{5}$.

Estas alteraciones tienen efectos aún más marcados en los animales de sistemas intensivos de producción, donde deben aprender a comer alimentos que difieren, en cuanto a su forma o presentación (como pellets o concentrados), de los encontrados en ambientes naturales ${ }^{12}$. Por su parte, la disponibilidad de superficie de comederos o momento de suministro del alimento, alteran el comportamiento ingestivo, observándose mermas en las ganancias de peso o productividad ${ }^{16}$. De esta manera, se deduce que modificar el ritmo de alimentación altera la homeostasis ${ }^{58}$ y por lo tanto el bienestar del individuo.

Ambiente social inter e intra especifico: en estado natural los animales no viven solos, cohabitan con otros individuos de su misma especie conformando grupos más o menos estables, dependiendo de la época del año y de la disponibilidad de recursos, entre otros ${ }^{17}$. Hay especies jerárquicas, otras organizadas a partir de un animal guía, otras que presentan combinaciones de ambas y otras que son cazadores solitarios. Un ejemplo lo constituyen los equinos que están organizados en matriarcados y es la yegua madrina la guía del grupo, en cambio en los bovinos y ovinos es indistinto, un macho o la hembra más experimentada ${ }^{43}$; mientras que los perros tienen una organización jerárquica no lineal que depende de la disponibilidad de recursos ${ }^{33}$. La conformación de los grupos en animales en cautiverio responde a la voluntad humana, lo que puede alterar las relaciones sociales naturalmente establecidas ${ }^{46}$.

Pero así como los individuos se relacionan con los de su misma especie (relación intra-específica), también lo hacen con otras especies (relación inter-específica). En este caso en particular deberíamos considerar las relaciones negativas: presa (bovino) y predador (canino), o competitivas como en el caso de dos predadores (perro y gato). El estrés está asociado a fallas en los procesos o estrategias adaptativas ${ }^{56}$. Debemos considerar que los procesos de control del comportamiento son cuatro: cognición, estímulos externos, realización del comportamiento y procesos de desvío o transición ${ }^{56}$. La percepción social y la cognición social son dos eventos diferentes que interactúan frente a los agentes estresantes ${ }^{3}$. Un bovino puede habituarse a un perro en particular, pero para su percepción, desde la especie a la que pertenece, ese perro siempre será un predador.

\section{RELACIÓN HUMANO ANIMAL}

Más allá de los procesos de domesticación que hubieran acompañado a las especies, la habituación a los seres humanos es un fenómeno complejo, en donde cabe destacar que la empatía juega un rol preponderante. Capacidad de amansamiento y docilidad son dos conceptos relacionados ${ }^{45}$. Mientras que la capacidad de amansamiento es un carácter heredable, la docilidad depende no sólo de los genes heredados sino también de la experiencia previa -fenotipo de esa docilidad-. La interacción positiva del animal con los seres humanos en edad temprana, mejora la expresión de la mansedumbre y por lo tanto disminuye su reactividad ${ }^{45}$. Deberíamos considerar que con su actitud cada individuo humano moldea la percepción que los animales tienen de la especie humana (macro escala -especie humana-) $y$, particularmente moldea el recuerdo que fijará un animal de ese individuo humano en particular, en base a la experiencia que haya experimentado con él (micro escala -hombre, mujer, niño, niña, anciano-). 
Dentro de las habilidades propias de los individuos humanos, que permiten una buena relación con los animales, podríamos destacar la capacidad de reconocer los comportamientos inherentes a cada especie, interactuando de acuerdo a las motivaciones de los animales en cuestión (base del manejo etológico). Por otra parte, la actitud de la persona hacia los animales está condicionada por miedo, ansiedad, autoconfianza, que son percibidos por los animales, modificando sus respuestas de comportamiento. Por ejemplo, la aproximación de un sujeto u objeto de gran tamaño en forma rápida podría ser interpretada como una señal más amenazante para el animal que si la aproximación fuera pequeñas y lenta ${ }^{45}$; otro ejemplo lo constituye la dirección de la mirada. Una mirada directa a los ojos puede representar un signo de amenaza o desafío. Nuestra propia postura corporal tiene significado; mientras que una aproximación agazapada es considerada como sumisión; la espalda hacia atrás es dominante, mientras que una espalda recta con los hombros hacia abajo representa una posición neutra ${ }^{42}$.

\section{CONCLUSIONES}

Si bien los animales domésticos se adaptaron a vivir en el ambiente antrópico, el hombre lo modifica continuamente. Muchas veces, persiguiendo un beneficio económico o estético, otras, en cambio, creyendo brindarles una mejor calidad de vida. Sin considerar si los motivos provienen de buenas o malas intenciones, estas modificaciones conllevan a un esfuerzo de readaptación continuo por parte de los animales, mediante diferentes métodos de aprendizaje cómo único medio para sobrevivir y adaptarse a estos ambientes tan inestables. Si bien el estrés agudo generado durante ese proceso puede asociarse con síntomas evidentes como diarrea, infecciones, alteración del ritmo de crecimiento y cambios de la conducta, cuando se presenta de manera crónica se manifiesta con alteraciones físicas del estado de salud, que pueden ser sutiles para el ojo no entrenado, pero que también comprometen el bienestar poniendo en riesgo la vida de los animales afectados.

Con frecuencia los agentes estresantes físicos son fáciles de reconocer, pero aquellos percibidos, los que por no ser reales no siempre se tienen en cuenta, son los que afectan al estado emocional de los animales, dando lugar al distrés ${ }^{35}$. Cuando se utilizan indicadores fisiológicos sin considerar los conductuales o ambientales, se puede incurrir en un error. Como ejemplo podemos mencionar la interpretación del nivel de cortisol en animales reproductivos, que tiene la misma elevación en la conducta de apareamiento que cuando están atados o confinados. La diferencia entre ambas situaciones está dada por los procesos cognitivos que desencadenan esas respuestas ${ }^{35}$.

Si la habilidad para aprender es considerada como una señal de la capacidad cognitiva y se presume que la cognición es un prerrequisito para sufrir ${ }^{39}$, reconocer el sufrimiento resulta indispensable para mejorar el bienestar. Dentro de los ejemplos de estados emocionales y mentales que conducen al sufrimiento en los animales domésticos tenemos desde el ayuno hasta las enfermedades. Pero no debemos olvidar a aquellos que nos resultan difíciles de detectar como la fatiga, dolor, miedo, irritación, depresión o aburrimiento que, sin embargo, son los que se presentan con mayor frecuencia ${ }^{39}$. Estas emociones son el resultado del confinamiento al que son sometidos tanto los animales de producción como los de compañía, por encontrarse en ambientes conformados artificialmente. Pensemos por ejemplo el contexto de sufrimiento que puede traer aparejado el encierro en un departamento, un canil o un corral.

Por otra parte, se reconoce que la habituación a la especie humana es indispensable para una buena relación humano-animal pero, que un animal haya logrado algún grado de socialización y no se observen reacciones asociadas a miedo, huida o escape, no quiere decir que lo cognitivo -en un contexto social- no se encuentre afectado. Probablemente, las personas que están en contacto diario con los animales, como los veterinarios prácticos, estén en una posición particularmente fuerte para reconocer el sufrimiento diario ${ }^{39}$. De esa manera, pueden resultar herramientas útiles para la educación de propietarios y productores con miras a incentivar modificaciones ambientales con intervenciones mínimas que permitan mejorar el bienestar, teniendo en cuenta las preferencias de cada especie.

Agradecimientos. Por su valiosa colaboración, a las integrantes del grupo Bienestar y Salud de la Facultad de Ciencias Veterinarias UNNE, particularmente a MV María Victoria Rossner, MV Romina Paola Cainzos y Lic. María Nieves Alabarcez.

\section{REFERENCIAS}

1. Abou-Ismail UA, Burman OH, Nicol CJ, Mendl M. 2008. Let sleeping rats lie: Does the timing of husbandry procedures affect laboratory rat behavior, physiology and welfare. Appl Anim Behav Sci 111: 329-341.

2. Accorsi PA, Carloni E, Valsecchi P, Vigiani R, Gamberoni M, Tamanini C. 2008. Cortisol determination in hair and faeces from domestic cats and dogs. Gen Comp Endocr 155: 398-402.

3. Adolphs R. 2001. The neurobiology of social cognition. Current Opin Neurobiol 11: 231-239.

4. Arteaga ML, Martínez M, Guevara R, Hudson R. 2007. Comunicación química en mamíferos domésticos. Vet Mex 38: 105-121.

5. Beaver V. 2003. Feline behavior. A guide for veterinarians, $2^{\text {nd }}$ ed., Saunders, Philadelphia, p. 215-225.

6. Beerda B, Scilder M, Janssen N, Mol J. 1996. The use of salivary cortisol, urinary cortisol, and catecholamine 
measurements for a noninvasive assessment of stress response in dogs. Horm \& Behav 30: 272-279.

7. Boissy A, Manteuffel G, Jensen MB, Moe RO, Spruijt B, Keeling LJ. 2007. Assessment of positive emotions in animals to improve their welfare. Physiol \& Behav 92: 375-397.

8. Broom DM, Johnson KG. 1993. Stress and animal welfare, Chapman \& Hall, London, $461 \mathrm{p}$.

9. Broom DM. 2001. Assessing the welfare of hens and broilers. Proc Aust Poult Sci Sym 13: 61-70.

10. Carlstead K, Brown JL, Strawon W. 1993. Behavioural and physiological correlated of stress in laboratory cats. Appl Anim Behav Sci 38: 143-158.

11. Casey R. 2002. Fear and stress in companion animals. In: Manual of canine and feline behavioural medicine (Horwitz D, Mills D, Heath S, ed.), Publ. BSAVA (British Small Animal Veterinary Association), London, p. 144-153.

12. Chapple RS, Wodzicka M, Lynch JJ. 1987. The learning behaviour of sheep when introduced to wheat. 1. Wheat acceptance of wheat and the effect of trough familiarity. Appl Anim Behav Sci 18: 157-162.

13. Dantzer R, Mormede P. 1983. Stress in farm animals: a need for reevaluation. J Anim Sci 57: 6-8.

14. Davenport MD, Tiefenbacher S, Lutz CK, Novak MA, Meyer JS. 2006. Analysis of endogenous cortisol concentrations in the hair of rhesus macaques. Gen \& Comp Endocr 147: 255-261.

15. Dawkins MS. 2006. Through animal eyes: what behaviour tells us. App Anim Behav Sci 100: 4-10.

16. De Vries TJ, Von Keyserlingk MA. 2006. Feed stalls affect the social and feeding behavior of lactating dairy cows. J Dairy Sci 89: 3522-3531.

17. Estevez I, Andersen IL, Naevdal E. 2007. Group size, density and social dynamics in farm animals. Appl Anim Behav Sci 103: 185-204.

18. Ewen BS, Chattarji S. 2007. Neuroendocrinology of stress. In: Handbook of neurochemistry and molecular neurobiology (Lajtha A, Mikoshiba K, Ed.), Springer, Berlin, p. 572-588.

19. Falewee C, Gautier E, Lafont $\mathbf{C}$, Bougrat L, Pageat $\mathbf{P}$. 2006. Effect of a synthetic equine maternal pheromone during a controlled fear-eliciting situation. Appl Anim Behav Sci 101: 144-153.

20. Frank DF, Erb HN, Houpt KA. 1999. Urine spraying in cats: presence of concurrent disease effects of a pheromone treatment. Appl Anim Behav Sci 61: 263-272.

21. Fraser AF. 1980. Comportamiento de los animales de granja, Acribia, Zaragoza, $291 \mathrm{p}$.

22. Fraser AF, Broom DM. 1997. Farm animal behavior and welfare, CABI, Wallingford, $368 \mathrm{p}$.

23. Gotoh M, Tajima T, Suzuki Y, Ikari H, Iguchi A, Kamuku S. 1998. Swimming stress that causes hyperglycemia increases in vivo release of noradrenaline, but not acetylcholine, from the hypothalamus of conscious rats. Brain Res 780: 74-79.

24. Goy I, Decosne C, Bonnet JM. 2007. Influence of aging on adrenal responsiveness in a population of eleven healthy beagles. Res Vet Sci 82: 195-201.
25. Guyton AC, Hall JE. 2006. Tratado de fisiología médica, $11^{\circ}$ ed., Elsevier, Madrid, 1038 p.

26. Henessy MB, Kaiser S, Sachser N. 2009. Social buffering of the stress response: diversity, mechanisms, and functions. Frontiers Neuroendocr 30: 470-482.

27. Kainuma E, Wantanabe M, Tomiyama C, Inoue $\mathbf{M}$, Kuwano Y, Ren H. 2009. Association of glucocorticoid with stress-induced modulation of body temperature, blood glucose and innate immunity. Psychoneuroendocr 34: 1459-1468.

28. Kirschbaum C, Hellhammer D. 1994. Salivary cortisol in psychoneuroendocrine. Psychoneuroendocr 19: $313-$ 333.

29. Kobelt AJ, Hemsworth PH, Barnett JL, Butler KL. 2003. Sources of sampling variation in saliva cortisol in dogs. Res Vet Sci 75: 157-161.

30. Kudielka BM, Hellhammer DH, Wust S. 2009. Why do we respond so differently? Reviewing determinants of human salivary cortisol responses to challenge. Psychoneuroencodocr 34: 2-18.

31. Lentfer TL, Gebhardt SG, Fröhlich EF, von Borell E. 2011. Influence of nest site on the behaviour of laying hens. Appl Anim Behav Sci 135: 70-77.

32. Manteca X. 2003. Etología clínica veterinaria del perro y del gato, $3^{\circ}$ Ed., Multimédica, Barcelona, p. 345 .

33. Miklosi A. 2007. Dog behaviour, evolution, and cognition. Oxford University Press, Oxford, p. 205-214.

34. Mills DS, Ramos D, Estelles MG. Hargrove C. 2006. A triple blind placebo controlled investigation into the assessment of the effect of dog appeasing pheromone (DAP) on anxiety related behaviour of problem dogs in veterinary clinic. Appl Anim Behav Sci 98: 114-126.

35. Moberg GP. 2000. Biological responses to stress: implications for animal welfare. In: The Biology of Animal Stress (Moberg GP, Mench JA, ed.), CABI Publishing, Oxford, p. $1-21$.

36. Mormede P, Andanson S, Auperin B, Beerda B, Guemene D, Malmkvist J. 2007. Exploration of the hypothalamic-pituitary-adrenal function as a tool to evaluate animal welfare. Physiol \& Behav 92: 317-339.

37. Morgan KN, Tromborg CT. 2007. Source of stress in captivity. Appl Anim Behav Scie 102: 263-302.

38. Nelson JR. 2005. An Introduction to Behavioral Endocrinology, $3^{\circ}$ ed., Sinauer Associates Inc., Massachusetts, p. 699-720.

39. Neville GG. 2004. Physiology and behavior of animal suffering. UFAW/Blackwell Animal Welfare Series, Oxford, p. 1-21.

40. Ohl F, Arndt SS, Staay FJ. 2008. Pathological anxiety in animals. Vet $J$ 175: 18-26.

41. Oitzl MS, Champagne DL, Veen R, Kloet ER. 2010. Brain development under stress: Hypotheses of glucocorticoids actions revisited. Neurosci \& Biobehav Rev 34: 853-866.

42. Pageat P. 1998. Patología del comportamiento del perro, Pulso Ediciones SA, Barcelona, p. 37.

43. Paranhos MJ. 2000. Ambiencia na produzao de bovino de corte a pasto. Anais Etol 18: 26-42. 
44. Price EO. 1999. Behavioural developments in animals undergoing domestication. Appl Anim Behav Sci 65: 245-271.

45. Price EO. 2002. Animal domestication and behaviour, CAB International, Wallington, p. 161.

46. Raussi S, Boissy A, Delval E, Pradel P, Kaihilahti J, Veissier I. 2005. Does repeated regrouping alter the social behavior of heifers? Appl Anim Behav Sci 93: 1-12.

47. Rochlitz I. 2005. A review of the housing requirements of domestic cats (Felis silvestris catus) kept in the home. Appl Anim Behav Sci 93: 97-109.

48. Rudloff E, Kirby R. 2001. Tratamiento del shock hipovolémico, Waltham Focus 11: 11-23.

49. Sapolsky RM, Romero LM, Munck AU. 2000. How do glucocorticoids influence stress responses? Integrating permissive, suppressive, stimulatory, and preparative actions. Endocr Rev 21: 55-89.

50. Selye H. 1976. The stress of life, McGraw-Hill, New York, p. 166 .

51. Sheppard G, Mills DS. 2003. Evaluation of dog appeasing pheromone as a potential treatment for dogs fearful of fireworks. Vet Rec 152: 432-436.

52. Sommerville BA, Broom DM. 1998. Olfactory awareness. Appl Anim Behav Sci 57: 269-286.

53. Stephen JM, Ledger RA. 2006. A longitudinal evaluation of urinary cortisol in kennel: Canis falimiliaris. Physiol \& Behav 87: 911-916.

54. Stoney CM, Finney M. 2007. Encyclopedia of Stress, $2^{\circ}$ ed., Academic Press, New York, p. 478-483.
55. Tilbrook AJ, Clarke IJ. 2006. Neuroendocrine mechanisms of innate status of attenuated responsiveness of the hypothalamo-pituitary adrenal axis to stress. Frontiers Neuroendocr 27: 285-307

56. Toates F. 2000. Multiple factors controlling behavior: Implications for stress and welfare. In: The Biology of Animal Stress (Moberg GP, Mench JA, ed.), CABI Publishing, Oxford, p. 199-226.

57. Tod E, Brander D, Waran N. 2005. Efficacy of dog appeasing pheromone in reducing stress and fear related behaviour in shelter dogs. Appl Anim Behav Sci 93: 295-308.

58. Ulrich YM, Herman JP. 2009. Neural regulation of endocrine and autonomic stress responses. Nat Rev Neurosci 10: 397-409.

59. Veissier I, Boissy A. 2006. Stress and welfare: Two complementary concepts that are intrinsically related to the animal's point of view. Phisiol \& Behav 92: 429-433.

60. Webster JI, Glaser R. 2008. Stress hormones and immune function. Cell Immunol 252: 16-26.

61. Wechsler B, Lea SE. 2007. Adaptation by learning: Its significance for farm animal husbandry. Appl Anim Behav Sci 108: 197-214.

62. Wells DL. 2009. Sensory stimulation as environmental enrichment for captive animals: A review. Appl Anim Behav Sci 118: 1-11.

63. Young EA, Abelson J, Lightman SL. 2004. Cortisol pulsatility and its role in stress regulation and health. Front Neuroendocr 5: 69-76.

\section{Asociación Cooperadora de la Facultad de Ciencias Veterinarias Universidad Nacional del Nordeste}

Personería Jurídica No 647/92 y 912/00

Sargento Cabral 2139

3400 Corrientes

Argentina

La Asociación Cooperadora de la Facultad de Ciencias Veterinarias de la UNNE fue constituida el 10 de diciembre del año 1991 como entidad de bien público, con el objeto de promover y coadyuvar las actividades científicas, educativas y culturales relacionadas con las ciencias veterinarias. En tal sentido, implementa acciones para colaborar con la enseñanza, extensión, actualización y difusión científica que realiza dicha casa de estudios.

\section{Beneficios que brinda a sus asociados:}

- Fotocopias con descuentos especiales del $20 \%$ en la Fotocopiadora Copias.com que funciona dentro del predio de la Facultad de Ciencias Veterinarias.

- Descuentos del 10\% para la adquisición de libros de la Editorial Inter-Médica.

- Descuentos especiales en otros rubros.

Sea solidario, asóciese en beneficio de toda la comunidad universitaria

Tel. 03783-425753 interno 186•Página Web www.vet.unne.edu.ar•E-mail: cooperadora@vet.unne.edu.ar 